\title{
Zeeman-split mesoscopic transport through a normal-metal-quantum-dot-superconductor system with ac response
}

\author{
Hong-Kang Zhao ${ }^{1,2, *}$ and Jian Wang ${ }^{1}$ \\ ${ }^{1}$ Department of Physics, The University of Hong Kong, Pokfulam Road, Hong Kong, China \\ ${ }^{2}$ Department of Physics, Beijing Institute of Technology, Beijing 100081, China \\ (Received 5 July 2000; revised manuscript received 12 March 2001; published 3 August 2001)
}

\begin{abstract}
We have investigated the Zeeman-split mesoscopic transport through the normal-metal-quantum-dotsuperconductor system applied with a microwave field. We employ BCS theory to describe the Hamiltonian of the superconducting lead. The time-averaged tunneling current formula is derived by using the Keldysh's nonequilibrium Green-function technique in the Nambu representation. The multilevel quantum dot is considered for the noninteraction system in the calculations. The spin split Andreev reflection and photon-electron pumping behaviors are investigated in the presence of a Zeeman magnetic field. The resonant structure versus Zeeman energy and gate voltage is revealed. The Zeeman-split photon-assisted $I-V$ characteristics are evaluated for the single-channel quantum-dot system. By adjusting the Zeeman magnetic field, we can obtain a large resonant tunneling current even if in the Andreev reflection regime. The current appears to have interesting structures versus Zeeman energy for the multichannel quantum-dot system associated with a different gate voltage. The negative and positive current appear in the symmetric forms by controlling the Zeeman field.
\end{abstract}

DOI: 10.1103/PhysRevB.64.094505

PACS number(s): 74.50.+r, 73.40.-c, 73.21.-b, 72.10.Bg

\section{INTRODUCTION}

Mesoscopic systems with small samples coupled to superconducting reservoirs play an important role in our investigation. There are two basic types for electrons transporting through a two-terminal structure in the presence of superconductivity: the superconductor-normal-metal-superconductor (SNS) junction with both electrodes being superconductors and the normal-metal-superconductor (NS) junction with one electrode a normal layer and one electrode a superconductor. Supercurrent can flow through the SNS system without any applied voltage, while we must apply a voltage for electric current to flow through the NS junction. The quasiparticles and electrons interact with one another and keep their phase memories during transport through the mesoscopic system to produce novel features. For example, the ultrasmall superconducting sample coupled with normal leads displays parity symmetry; ${ }^{1}$ the even-odd parity asymmetry and the Coulomb blockade of Andreev reflection ${ }^{2}$ are found in such systems. For SNS or NS systems, most of the investigations in the literature deal with ballist transport by using scattering theory. ${ }^{3-6}$ The quasiparticles are scattered at the NS junctions. If the source-drain voltage between a junction is smaller than the energy gap of the superconductor, i.e., $|e V|<|\Delta|$, there also exists small current due to Andreev reflection. ${ }^{7}$ The noninteracting scattering theory corresponds to the usual Landauer-Büttiker scattering theory in normal samples. For superconducting leads coupled with junctions, the quasiparticles are dominated either by time-independent or by time-dependent Bogoliubov-de Gennes equations..$^{7-12}$ Under the influence of source-drain voltage, a quasiparticle trajectory in the clean normal region of a SNS junction has been described by an accelerated wave packet of the particle. ${ }^{9,13}$

Recently, there has been great interest to study the systems of superconducting leads coupled with quantum-dot systems, for instance, the investigation of the tunneling through the normal quantum dot connected with superconducting leads. The systems as superconductor-quantumdot-superconductor (SDS), superconductor-quantum-dotnormal-lead (SDN), and superconducting quantum dot connected with normal leads possess very rich physical phenomena. These systems can be used as the models of quantum devices coupled with superconducting leads. Since the superconducting and normal leads are much larger than the quantum dots, they are treated as the equilibrium reservoirs. The quasiparticles and electrons are emitted from one of the reservoirs and then are scattered by the central region. Since the sizes of quantum dots are smaller than the phasecoherence lengths, the quasiparticles and electrons keep phase memories in the quantum-dot region. Some interesting properties arise from the coherent transports. However, for the system with superconductors coupled with the quantum dot, the transport problems are very complicated due to multiple scatterings of quasiparticles and electrons in the central quantum dot. The nonlinear transport contains many significant structures which remain to be discovered. For such hybrid systems, the quantum effects are distinct because the quasiparticles can keep phase memories for a considerable distance, and the electron energy of the quantum dot becomes discrete as the quantum dot is sufficiently small. There may exist resonant structure behaviors in the tunneling currents. Analogous to the normal sample systems, we can impose microwave fields to the hybrid systems. The electrons and quasiparticles absorb and emit photons during transport to form split resonant structures. Obviously, the tunneling current possesses compound effects associated with superconducting leads and the quantum dot. For the ultrasmall quantum dot, the density of state (DOS) of quasiparticles in the superconducting leads is obtained by measuring the $I-V$ characteristics of the SDS system. ${ }^{14}$ The experimental observation of a signature of phase-coherent Andreev reflection is reported by Lenssen et al. through studying the 
system of superconducting contacts connected with twodimensional electron gas in $\mathrm{GaAs} / \mathrm{Al}_{x} \mathrm{Ga}_{1-x} \mathrm{As}$ heterostructures. ${ }^{15}$ The corresponding theoretical work contributing to the noninteracting systems has been performed by Claughton et al. ${ }^{16}$ by using the Green-function technique. We have investigated the photon-assisted tunneling through the SDN system where the Coulomb interaction is not considered, and the electron-photon pumping effect has been found. The spin split Andreev reflection and $I-V$ characteristics and the spin nondegenerated dc Josephson current oscillation also have been investigated. ${ }^{17}$ The multiple discrete level Andreev reflection is discussed in the SDN system, and different kinds of resonant peaks in the current versus gate voltage are discovered. ${ }^{18}$

As electrons in a system are exposed to an external magnetic field, the energy of an electron is split due to the Zeeman effect. The tunneling current in a lead is certainly affected by the Zeeman magnetic field, and the magnetic moment of electrons plays an important role in transport. The spin degenerated electron system becomes a spin nondegenerated one. As the magnetic field $\mathbf{B}$ is small enough, the electron energy is split by adding the Zeeman energy $\sigma \mu B$ to the original energy level, where $\sigma$ is the spin value, and $\mu$ is the magnetic moment of the electron. In this paper we consider the normal-metal-quantum-dot-superconductor (NDS) system with a Zeeman magnetic field applying to the quantum dot. Because the quantum dot is a kind of quantum device, we have several methods to control the tunneling current. We can impose a dc source-drain voltage between the leads and apply a voltage through the gate on the quantum dot. The gate voltage adjusts the energy levels of the quantum dot. On the other hand, in the quantum devices we often encounter the situation in which they are irradiated by microwave fields. The tunneling current is modulated by the external signals, and the output current is sensitively relying on it. We consider the problem with a Zeeman magnetic field applying to the quantum dot through the gate. The magnetic field is screened so as not to affect the superconducting and normal leads. The source and drain of the system are biased by a dc voltage and an ac microwave field. The quantum dot is applied by a dc gate voltage to control the tunneling current. We derive the tunneling current formula by employing the Keldysh's nonequilibrium Green-function (NGF) technique, and perform the numerical calculations at zero temperature for the single-channel and multichannel quantumdot systems. The tunneling resonant structures versus gate voltage and Zeeman magnetic field are obtained, and tunneling behaviors caused by the Zeeman magnetic field are observed. The $I-V$ characteristics of the single-channel system are presented to show the Zeeman-split Andreev tunneling and photon-electron pumping behaviors. The remainder of this paper is organized as follows. Section II is devoted to derive the tunneling current by employing the Keldysh's NGF technique. Section III is arranged to perform the numerical calculations on the tunneling current for the singlechannel and multichannel systems. The concluding remarks are given in Sec. IV.

\section{HAMILTONIAN AND FORMALISM}

The system is composed of three parts: the left normalmetal lead, the right superconducting lead, and the quantum dot. We consider the circumstance where the two leads are biased by the dc voltage $V$ which is the drop of chemical potentials between two leads $\mu_{R}-\mu_{L}=e V$. A magnetic field $\mathbf{B}$ is applied to the quantum dot through the gate. This magnetic field is screened in order not to affect the superconducting and normal leads. A microwave field with frequency $\omega$ is imposed to the system forming a potential drop $e \widetilde{V}_{R L} \cos (\omega t)$ between the two leads, where we have taken the potential of the left lead as a reference for measurement. This ac potential drop is related to the dipole approximation. So the electron energy in the $\gamma$ th lead is described by the timedependent one $\varepsilon_{\gamma, k \sigma}(t)=\epsilon_{\gamma, k \sigma}+e \widetilde{V}_{\gamma L} \cos (\omega t)$, where $\epsilon_{\gamma, k \sigma}$ $=E_{\gamma, k \sigma}-\mu_{\gamma}, \widetilde{V}_{\gamma L}=\widetilde{V}_{\gamma}-\widetilde{V}_{L}$. The energy of electrons in the quantum dot is defined by $\widetilde{E}_{d, l \sigma}-\sigma \mu B$, where $\widetilde{E}_{d, l \sigma}=E_{d, l \sigma}$ $-e v_{g}, E_{d, l \sigma}$ is the energy level of the quantum dot in the presence of the gate voltage $v_{g}$. The gate voltage and the Zeeman energy $\mu B$ adjust the quantum-dot energy. $\mu$ $=g \mu_{B} / 2$ is the magnetic moment of electrons, where $\mu_{B}$ is the Bohr magneton, and $\mu_{B} \approx 0.5788 \times 10^{-15} \mathrm{meV} / \mathrm{G}$. The $g$ factor is equal to 2.003 for a free-electron system. The two leads are in macroscopic sizes, which means that the two leads in three dimensions are larger than the coherence lengths of quasiparticles and tunneling electrons. So the particles in leads can be treated by using the grand canonical ensemble theory in the equilibrium state. We employ the BCS theory to deal with the transport of electrons in the superconducting lead. The Hamiltonian of the superconducting lead is approximated as the usual mean-field theory. The normal metal is described by the free-electron gas system. The quantum dot is considered as the noninteracting model. Therefore, the Hamiltonian of the system can be expressed by the sum of three separate sub-Hamiltonians and tunneling terms as

$$
\begin{aligned}
H= & \sum_{k \sigma} \varepsilon_{R, k \sigma}(t) a_{R, k \sigma}^{\dagger} a_{R, k \sigma} \\
& -\sum_{k}\left[\Delta a_{R, k \uparrow}^{\dagger} a_{R,-k \downarrow}^{\dagger}+\Delta * a_{R,-k \downarrow} a_{R, k \uparrow}\right] \\
& +\sum_{k \sigma} \epsilon_{L, k \sigma} a_{L, k \sigma}^{\dagger} a_{L, k \sigma}+\sum_{\sigma l}\left(\widetilde{E}_{d, l \sigma}-\sigma \mu B\right) d_{l \sigma}^{\dagger} d_{l \sigma} \\
& +\sum_{k l \sigma \gamma}\left(T_{\gamma k} a_{\gamma, k \sigma}^{\dagger} d_{l \sigma}+\text { H.c. }\right)
\end{aligned}
$$

where $a_{\gamma, k \sigma}^{\dagger}\left(a_{\gamma, k \sigma}\right)$ and $d_{l \sigma}^{\dagger}\left(d_{l \sigma}\right)$ are the creation (annihilation) operators of electrons in the two leads and quantum dot, respectively. $\Delta$ is the energy gap of the superconducting lead defined by $\Delta=V_{R} \Sigma_{k}\left\langle a_{R,-k \downarrow} a_{R, k \uparrow}\right\rangle$. Its conjugate is defined by $\Delta^{*}=V_{R} \Sigma_{k}\left\langle a_{R, k \uparrow}^{\dagger} a_{R,-k \downarrow}^{\dagger}\right\rangle$. The energy gap is assumed to be known, and it is considered to be the one without cou- 
pling. $T_{\gamma k}$ is the interaction strength of electrons between the $\gamma$ th lead and quantum dot. It is complex and satisfies the relation $T_{\gamma k}=T_{\gamma-k}$. We have taken the chemical potential of the left lead as the reference of energy measurement. The spin $\sigma$ has the values of $\sigma=+1$ and -1 corresponding to the notations $\uparrow$ and $\downarrow$, respectively, in the subscripts of equations. We make gauge transformation to change the timedependent energy into a time-independent one. This transformation is settled by changing the interaction strengths to be time dependent. So the time-independent interaction strength of the $\gamma$ th lead acquires a time-dependent phase factor, i.e., $T_{\gamma k} \rightarrow \widetilde{T}_{\gamma k}(t)$ by the gauge transformation, where

$$
\widetilde{T}_{\gamma k}(t)=T_{\gamma k} \exp \left[-\frac{i}{\hbar}\left(\mu_{\gamma}-\mu_{L}\right) t+i \Lambda_{\gamma L} \sin (\omega t)\right],
$$

and $\Lambda_{\gamma L}=\left(e \widetilde{V}_{\gamma L}\right) / \hbar \omega$. In fact, the gauge transformation only makes the interaction strength of right lead to be a timedependent one.

The tunneling current in the $\gamma$ th lead transporting into the quantum dot can be formulated by using the continuity equation and the Heisenberg equation. As a consequence we can derive the current by

$$
I_{\gamma}(t)=-\frac{i e}{\hbar} \sum_{k \sigma}\left\langle\left[H, a_{\gamma, k \sigma}^{\dagger}(t) a_{\gamma, k \sigma}(t)\right]\right\rangle,
$$

where $\langle\cdots\rangle$ is the notation of the quantum expectation value and ensemble average. Substituting the Hamiltonian of the system into the current formula (3), one can find the timedependent current formula determined by the correlation functions of electrons between the leads and the quantum dot. These correlation functions can be expressed by NGF's of the quantum dot.

In order to perform the calculation analogous to the technique used by treating the system coupled with normal leads, we make the Bogoliubov transformation to diagonalize the superconducting lead. ${ }^{19}$ The Hamiltonian of the superconducting lead is expressed as the diagonal form $H_{R}$ $=\Sigma_{k \sigma} \xi_{R k} \alpha_{R, k \sigma}^{\dagger} \alpha_{R, k \sigma}$, where $\alpha_{R, k \sigma}^{\dagger}$ and $\alpha_{R, k \sigma}$ are the creation and annihilation operators of the quasiparticle in the superconducting lead, and they satisfy the Fermi distribution. $\xi_{R k}$ is the excitation energy of the quasiparticle. We consider the case where the energy gap is given by $\Delta=|\Delta| e^{i \phi}$. To proceed, we define the retarded (advanced) Green functions in the Nambu representation as

$$
\mathbf{G}_{l l^{\prime} \sigma}^{r(a)}\left(t, t^{\prime}\right)=\left(\begin{array}{cc}
\left\langle\left\langle d_{l \sigma}(t), d_{l^{\prime} \sigma}^{\dagger}\left(t^{\prime}\right)\right\rangle\right\rangle^{r(a)}, & \left\langle\left\langle d_{l \sigma}(t), d_{l^{\prime}-\sigma}\left(t^{\prime}\right)\right\rangle\right\rangle^{r(a)} \\
\left\langle\left\langle d_{l-\sigma}^{\dagger}(t), d_{l^{\prime} \sigma}^{\dagger}\left(t^{\prime}\right)\right\rangle\right\rangle^{r(a)}, & \left\langle\left\langle d_{l-\sigma}^{\dagger}(t), d_{l^{\prime}-\sigma}\left(t^{\prime}\right)\right\rangle\right\rangle^{r(a)}
\end{array}\right),
$$

where

$$
\left\langle\left\langle A(t), B\left(t^{\prime}\right)\right\rangle\right\rangle^{r(a)}=\mp(i / \hbar) \theta\left( \pm t \mp t^{\prime}\right)\left\langle\left[A(t), B\left(t^{\prime}\right)\right]_{+}\right\rangle .
$$

The Keldysh Green function in the Nambu representation is defined as

$\mathbf{G}_{l l^{\prime} \sigma}^{<}\left(t, t^{\prime}\right)=\frac{i}{\hbar}\left(\begin{array}{cc}\left\langle d_{l^{\prime} \sigma}^{\dagger}\left(t^{\prime}\right) d_{l \sigma}(t)\right\rangle, & \left\langle d_{l^{\prime}-\sigma}\left(t^{\prime}\right) d_{l \sigma}(t)\right\rangle \\ \left\langle d_{l^{\prime} \sigma}^{\dagger}\left(t^{\prime}\right) d_{l-\sigma}^{\dagger}(t)\right\rangle, & \left\langle d_{l^{\prime}-\sigma}\left(t^{\prime}\right) d_{l-\sigma}^{\dagger}(t)\right\rangle\end{array}\right)$.

From Eq. (3) we obtain the current formula in the $\gamma$ th lead as

$$
\begin{aligned}
I_{\gamma}(t)= & 2 e \operatorname{Re} \sum_{l l^{\prime} \sigma} \int d t_{1}\left[\mathbf{G}_{l l^{\prime} \sigma}^{r}\left(t, t_{1}\right) \widetilde{\boldsymbol{\Sigma}}_{\gamma \sigma}^{<}\left(t_{1}, t\right)\right. \\
& \left.+\mathbf{G}_{l l^{\prime} \sigma}^{<}\left(t, t_{1}\right) \widetilde{\boldsymbol{\Sigma}}_{\gamma \sigma}^{a}\left(t_{1}, t\right)\right]_{11},
\end{aligned}
$$

where $\widetilde{\boldsymbol{\Sigma}}_{\gamma \sigma}^{X}\left(t, t^{\prime}\right), X \in\{r, a,<\}$ denotes the self-energy matrix of the $\gamma$ th lead. This self-energy matrix describes the free quasiparticles or electrons in the $\gamma$ th lead interacting with the central quantum dot through the interaction strength $\widetilde{T}_{\gamma k}(t)$. In the current formula, we have to take the sum over $k$. This summation can be changed into an integral by introducing the linewidth $\Gamma_{\gamma \sigma}(E)=2 \pi \Sigma_{k}\left|T_{\gamma k}\right|^{2} \delta\left(E-E_{\gamma, k \sigma}\right)$ into the formula. In the wideband limit approximation, the linewidth is energy independent, i.e., $\Gamma_{\gamma \sigma}(E)=\Gamma_{\gamma \sigma}$. Since the energy $E_{\gamma, k \sigma}$ is a spin degenerated one, the linewidth is independent on the spin variable $\sigma$. In what follows, we neglect the spin subscript $\sigma$ in the linewidth by letting $\Gamma_{\gamma \sigma}$ $=\Gamma_{\gamma}$. The self-energy matrices $\widetilde{\boldsymbol{\Sigma}}_{\gamma \sigma}^{r(a)}\left(t, t^{\prime}\right)$ and $\widetilde{\boldsymbol{\Sigma}}_{\gamma \sigma}^{<}\left(t, t^{\prime}\right)$ can be expressed by the Green functions of quasiparticles and electrons in the leads. In the wideband limit, these selfenergy matrices take the following forms:

$$
\begin{aligned}
\widetilde{\mathbf{\Sigma}}_{\gamma \sigma}^{r}\left(t, t^{\prime}\right)= & -\frac{i}{h} \theta\left(t-t^{\prime}\right) \Gamma_{\gamma} \sum_{m n} J_{m}\left(\Lambda_{\gamma L}\right) J_{n}\left(\Lambda_{\gamma L}\right) \\
& \times \int d \epsilon N_{\gamma}(\epsilon) e^{-(i / \hbar) \epsilon\left(t-t^{\prime}\right)} \mathbf{\Sigma}_{\gamma \sigma}^{(0) m n}\left(t, t^{\prime}\right), \\
\widetilde{\mathbf{\Sigma}}_{\gamma \sigma}^{<}\left(t, t^{\prime}\right)= & \frac{i}{h} \Gamma_{\gamma} \sum_{m n} J_{m}\left(\Lambda_{\gamma L}\right) J_{n}\left(\Lambda_{\gamma L}\right) \\
& \times \int d \epsilon N_{\gamma}(\epsilon) e^{-(i / \hbar) \epsilon\left(t-t^{\prime}\right)} f(\epsilon) \mathbf{\Sigma}_{\gamma \sigma}^{(0) m n}\left(t, t^{\prime}\right),
\end{aligned}
$$

where 


$$
\begin{aligned}
& \boldsymbol{\Sigma}_{L \sigma}^{(0) m n}\left(t, t^{\prime}\right)=\left(\begin{array}{cc}
e^{-i\left(m t-n t^{\prime}\right) \omega} & 0 \\
0 & e^{i\left(m t-n t^{\prime}\right) \omega}
\end{array}\right) \\
& \boldsymbol{\Sigma}_{R \sigma}^{(0) m n}\left(t, t^{\prime}\right)=\left(\begin{array}{cc}
e^{i / \hbar\left[e V\left(t-t^{\prime}\right)-\left(m t-n t^{\prime}\right) \hbar \omega\right]}, & \frac{\Delta}{|\epsilon|} e^{i / \hbar\left[e V\left(t+t^{\prime}\right)-\left(m t+n t^{\prime}\right) \hbar \omega\right]} \\
\frac{\Delta^{*}}{|\epsilon|} e^{-i / \hbar\left[e V\left(t+t^{\prime}\right)-\left(m t+n t^{\prime}\right) \hbar \omega\right]}, & e^{-i / \hbar\left[e V\left(t-t^{\prime}\right)-\left(m t-n t^{\prime}\right) \hbar \omega\right]}
\end{array}\right) .
\end{aligned}
$$

The advanced self-energy matrix $\widetilde{\boldsymbol{\Sigma}}_{\gamma \sigma}^{a}\left(t, t^{\prime}\right)$ is taken on the similar form as $\widetilde{\boldsymbol{\Sigma}}_{\gamma \sigma}^{r}\left(t, t^{\prime}\right), f(\boldsymbol{\epsilon})$ is the Fermi distribution function defined by

$$
f(\epsilon)=\frac{1}{\exp \left(\epsilon / k_{B} T\right)+1},
$$

and $N_{\gamma}(\epsilon)$ is the DOS of the $\gamma$ th lead. For the superconducting lead we have

$$
N_{R}(\epsilon)=\frac{|\epsilon|}{\left(\epsilon^{2}-|\Delta|^{2}\right)^{1 / 2}},
$$

which is a complex in the Andreev reflection regime, and for the normal lead, we have $N_{L}(\epsilon)=1 . J_{m}(\lambda)$ are the Bessel functions of the first kind. The momentum of a quasiparticle may possess a small imaginary value. This means that the quasiparticle can penetrate through the barrier of the NS junction even if $0<|e V|<|\Delta|$ by means of the Andreev reflection. So there exists a small quantity of tunneling current in the leads caused by the Andreev reflection.

The current formula (6) is reduced to the form expressed by the Green functions of the quantum dot. Since we are interested in the time-averaged transport problems which may be obtained by experiments, we only need to know the time-averaged tunneling current in one lead due to the current conservation $I_{L}+I_{R}=0$. We derive the time-dependent current formula in the left lead by taking $\gamma=L$ in Eq. (6) and deduce it to the current formula similar to the normal system presented by Jauho, Wingreen, and Meir in Ref. 20 as

$$
\begin{aligned}
I_{L}(t)= & -\frac{2 e}{h} \operatorname{Im} \sum_{l l^{\prime} \sigma} \int_{-\infty}^{t} d t_{1} \int d \epsilon \Gamma_{L} e^{-(i / \hbar) \epsilon\left(t_{1}-t\right)} \\
& \times\left[f(\epsilon) G_{l l^{\prime} \sigma, 11}^{r}\left(t, t_{1}\right)+G_{l l^{\prime} \sigma, 11}^{<}\left(t, t_{1}\right)\right] .
\end{aligned}
$$

Since the system is perturbed by the microwave field, the tunneling current is characterized with the oscillating features of the external microwave field. The current evolves with time, and the time-reversal symmetry is broken. The Green functions of the quantum dot $G_{l l^{\prime} \sigma, 11}^{X}\left(t, t^{\prime}\right)$ cannot be described by the time-difference form because of the ac perturbation. The quantum dot provides multichannels for electrons to transport. The external microwave field splits the channels to form sidebands, and the Zeeman field splits the energy levels further. Therefore, the current is composed of infinite components of oscillating current tunneling through the quantum dot in the infinite channels. We derive the current formula directly from Eq. (9).

In order to find the Green functions of the quantum dot, we employ the equation of motion (EOM) method. We define the Green function $\mathbf{g}_{l l^{\prime} \sigma}^{r(a)}\left(t, t^{\prime}\right)$ as the diagonal matrix form in the derivation

$$
\begin{aligned}
& \mathbf{g}_{l l^{\prime} \sigma}^{r(a)}\left(t, t^{\prime}\right)=\mp \frac{i}{\hbar} \theta\left( \pm t \mp t^{\prime}\right) \\
& \times\left(\begin{array}{cc}
e^{-i / \hbar\left(\widetilde{E}_{d, l}-\sigma \mu B\right)\left(t-t^{\prime}\right)}, & 0 \\
0 & e^{i / \hbar\left(\widetilde{E}_{d, l}+\sigma \mu B\right)\left(t-t^{\prime}\right)}
\end{array}\right) \\
& \times \delta_{l, l^{\prime}} \text {. }
\end{aligned}
$$

This Green function describes the local electrons in the isolated quantum dot applied with the Zeeman field. Since the system is a coupled one, the self-energy of the quantum dot is associated with leads. For the superconductor connected quantum-dot system, the coupling becomes very complicated resulting from multiple reflections at the NS barriers. To find $\mathbf{G}_{l l^{\prime} \sigma}^{r(a)}\left(t, t^{\prime}\right)$, one has to find the self-energies of electrons in the quantum dot and then solve the equation chain selfconsistently. In fact, the self-energies of the quantum dot are equal to the ones given in Eqs. (7) and (8). The EOM can be reduced to the following Dyson equation:

$$
\begin{aligned}
\mathbf{G}_{l l^{\prime} \sigma}^{r(a)}\left(t, t^{\prime}\right)= & \mathbf{g}_{l l^{\prime} \sigma}^{r(a)}\left(t, t^{\prime}\right)+\sum_{l^{\prime \prime}} \iint d t_{1} d t_{2} \mathbf{g}_{l l \sigma}^{r(a)}\left(t, t_{1}\right) \\
& \times \widetilde{\boldsymbol{\Sigma}}_{l \sigma}^{r(a)}\left(t_{1}, t_{2}\right) \mathbf{G}_{l^{\prime \prime} l^{\prime} \sigma}^{r(a)}\left(t_{2}, t^{\prime}\right) .
\end{aligned}
$$

Equation (11) has to be solved self-consistently by iteration. In the presence of the Zeeman field, the occupation number is spin nondegenerated. This means that the electrons occupy the quantum dot differently for the spin-up and spin-down states. In the similar procedure, we can derive the Keldysh Green function $\mathbf{G}_{l l^{\prime} \sigma}^{<}\left(t, t^{\prime}\right)$ by employing the EOM and obtain the integral equation 


$$
\begin{aligned}
& \mathbf{G}_{l l^{\prime} \sigma}^{<}\left(t, t^{\prime}\right)=\sum_{l^{\prime \prime}} \iint d t_{1} d t_{2} \mathbf{g}_{l l \sigma}^{r}\left(t, t_{1}\right) \\
& \quad \times\left[\widetilde{\boldsymbol{\Sigma}}_{l \sigma}^{r}\left(t_{1}, t_{2}\right) \mathbf{G}_{l^{\prime \prime} l^{\prime} \sigma}^{<}\left(t_{2}, t^{\prime}\right)+\tilde{\boldsymbol{\Sigma}}_{l \sigma}^{<}\left(t_{1}, t_{2}\right) \mathbf{G}_{l^{\prime \prime} l_{\sigma}^{\prime}}^{a}\left(t_{2}, t^{\prime}\right)\right] .
\end{aligned}
$$

For the system with a quantum dot coupled to normal leads, the integral Eq. (12) gives the same result presented in Ref. 20. However, for the quantum dot coupled with superconducting and normal leads, this equation provides more information originating from the scattering procedure of the superconductor.

Equations (11) and (12) determine all the Green functions of the quantum dot. Each of the matrix equations above contains four equations of the Green function, and the Green functions are connected to each other in the equations. This requires us to solve the equations consistently. Therefore, from Eqs. (11) and (12), we can find the Green functions of the quantum dot and then arrive at the tunneling current from Eq. (9). Since we are interested in the time-averaged tunneling current, we only need to calculate the Green functions related to the Fourier transformed versions in diagonal variable forms. We make the Fourier transformation over Eqs. (11) and (12) versus the two times $t$ and $t^{\prime}$, then obtain algebraic equations associated with these Green functions. We define the quantity $\kappa_{l \sigma}^{ \pm}(E)$ as

$$
\kappa_{l \sigma}^{ \pm}(E)=\frac{1}{E \pm \widetilde{E}_{d, l \sigma}+\sigma \mu B}
$$

and define the retarded self-energy matrices in the Fourier transformed version as

$$
\boldsymbol{\Sigma}_{L m}^{r}(E)=-\frac{i}{2} \Gamma_{L}\left(\begin{array}{ll}
1, & 0 \\
0 & 1
\end{array}\right)
$$

for the left lead and

$$
\boldsymbol{\Sigma}_{R m}^{r}(E)=-\frac{i}{2} \Gamma_{R}\left(\begin{array}{cc}
N_{R}\left(E_{m}^{-}\right), & \Delta \tilde{N}_{R}\left(E_{m}^{-}\right) \\
\Delta * \tilde{N}_{R}\left(E_{m}^{+}\right), & N_{R}\left(E_{m}^{+}\right)
\end{array}\right)
$$

for the right lead, where $E_{m}^{ \pm}=E \pm(m \hbar \omega-e V), \widetilde{N}_{R}(E)$ $=N_{R}(E) /|E|$. In the self-energy of the right lead, the DOS of the superconductor plays an important role. The electrons are accelerated by the source-drain voltage as well as by the microwave field to form the energy sideband $E \pm(m \hbar \omega$ $-e V$ ), which causes a complicated Andreev reflection in the tunneling problems. We define the quantity $Q_{\gamma l m, \alpha \beta}^{( \pm) X}(E)$ to express Green functions in the derivation as

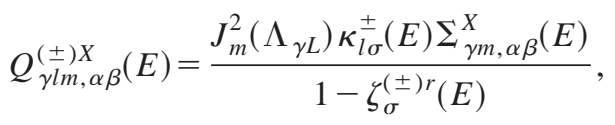

where

$$
\zeta_{\sigma}^{(+) r}(E)=\sum_{\gamma m l} J_{m}^{2}\left(\Lambda_{\gamma L}\right) \kappa_{l \sigma}^{+}(E) \Sigma_{\gamma m, 22}^{r}(E)
$$

$$
\zeta_{\sigma}^{(-) r}(E)=\sum_{\gamma m l} J_{m}^{2}\left(\Lambda_{\gamma L}\right) \kappa_{l \sigma}^{-}(E) \Sigma_{\gamma m, 11}^{r}(E) .
$$

Substituting the corresponding elements of Eqs. (13) and (14) into the definition of $\zeta_{\sigma}^{( \pm) r}(E)$, we can write them as the concrete forms

$$
\zeta_{\sigma}^{( \pm) r}(\epsilon)=-\frac{i}{2} \Lambda_{\sigma}^{( \pm)}(\epsilon) \tau^{( \pm)}(\epsilon)
$$

where

$$
\tau^{( \pm)}(\epsilon)=\Gamma_{L}+\Gamma_{R} \sum_{m} J_{m}^{2}\left(\Lambda_{R L}\right) N_{R}[\epsilon \pm(m \hbar \omega-e V)]
$$

and $\quad \Lambda_{\sigma}^{( \pm)}(\epsilon)=\Sigma_{l} \kappa_{l \sigma}^{ \pm}(\epsilon)$. As $\quad|\epsilon \pm(m \hbar \omega-e V)|>|\Delta|$, $\tau^{( \pm)}(\epsilon)$ is real, and as $|\epsilon \pm(m \hbar \omega-e V)|<|\Delta|, \tau^{( \pm)}(\epsilon)$ is a complex quantity.

We define the Green function $G_{\sigma, \alpha \beta}^{X}(E)$ by taking the summation of the Green function $G_{l l^{\prime} \sigma, \alpha \beta}^{X}(E)$ over all the channels of the quantum dot, i.e., $G_{\sigma, \alpha \beta}^{X}(E)$ $=\Sigma_{l l^{\prime}} G_{l l^{\prime} \sigma, \alpha \beta}^{X}(E)$. From Eq. (11) we find the retarded Green function $G_{\sigma, 11}^{r}(E)$ in the Fourier transformed version as

$$
G_{\sigma, 11}^{r}(E)=\frac{\Lambda_{\sigma}^{(-)}(E)}{1-\zeta_{\sigma}^{(-) r}(E)-\Pi_{\sigma}^{r}(E)},
$$

where

$$
\begin{aligned}
\Pi_{\sigma}^{r}(E)= & \sum_{l l^{\prime} \gamma \gamma^{\prime} m} J_{m}^{2}\left(\Lambda_{\gamma L}\right) \kappa_{l \sigma}^{-}(E) \Sigma_{\gamma m, 12}^{r}(E) \\
& \times Q_{\gamma^{\prime} l^{\prime} m+\left[\mu_{\gamma^{\prime} \gamma} / \hbar \omega\right], 21}^{(+))}\left(E+2 \mu_{\gamma L}-2 m \hbar \omega\right) .
\end{aligned}
$$

This Green function describes the resonant structure of electrons in the quantum dot. If electron energy in the channel $l$ is located in the normal region, the system resonates at $E$ $=\widetilde{E}_{l, d}-\sigma \mu B$. As the electron energy is in the Andreev reflection region, the system resonates at new levels. Equation (15) is the retarded Green function for the system in a pseudoequilibrium state. It can be used to calculate the timeaveraged tunneling current. The advanced Green function $G_{\sigma, 11}^{a}(E)$ can be derived similarly. For the pseudoequilibrium state, the advanced Green function is the conjugate of the retarded Green function, i.e., $G_{\sigma, 11}^{a}(E)=\left[G_{\sigma, 11}^{r}(E)\right]^{*}$.

We define the matrix $\boldsymbol{\Sigma}_{\gamma m}^{<}(E)$ associated with the Fourier transformed Keldysh self-energy as

$$
\boldsymbol{\Sigma}_{L m}^{<}(E)=i \Gamma_{L}\left(\begin{array}{cc}
f\left(\widetilde{E}_{m}^{-}\right), & 0 \\
0, & f\left(\widetilde{E}_{m}^{+}\right)
\end{array}\right)
$$

for the left lead, where $\widetilde{E}_{m}^{ \pm}=E \pm m \hbar \omega$, and

$$
\boldsymbol{\Sigma}_{R m}^{<}(E)=i \Gamma_{R}\left(\begin{array}{cc}
N_{R}\left(E_{m}^{-}\right) f\left(E_{m}^{-}\right), & \Delta \tilde{N}_{R}\left(E_{m}^{-}\right) f\left(E_{m}^{-}\right) \\
\Delta^{*} \widetilde{N}_{R}\left(E_{m}^{+}\right) f\left(E_{m}^{+}\right), & N_{R}\left(E_{m}^{+}\right) f\left(E_{m}^{+}\right)
\end{array}\right)
$$


for the right lead. The notation $E_{m}^{ \pm}$has the same expression shown in Eq. (14). The self-energies given in Eqs. (16) and (17) combine the features of charge carriers in the leads and the information of the quantum dot. This signifies that the distribution of electrons in the quantum dot is affected by the two leads. The temperature of the system influences the tunneling behaviors through the Fermi distribution function. In fact, the tunneling property is quite different for the zeroand nonzero-temperature systems.

From Eq. (12), one obtains the normal Keldysh Green function $G_{\sigma, 11}^{<}(E)$ in the pseudoequilibrium state as

$$
G_{\sigma, 11}^{<}(E)=G_{\sigma, 11}^{r}(E) \Pi_{\sigma}^{<}(E) G_{\sigma, 11}^{a}(E),
$$

where

$$
\begin{aligned}
\Pi_{\sigma}^{<}(E)= & \frac{1}{\Lambda_{\sigma}^{(-)}(E)} \sum_{\gamma l m} J_{m}^{2}\left(\Lambda_{\gamma L}\right) \kappa_{l \sigma}^{-}(E)\left[\Sigma_{\gamma m, 11}^{<}(E)\right. \\
& \left.+M_{\gamma m}\left(E, E+2 \mu_{\gamma L}-2 m \hbar \omega\right)\right], \\
M_{\gamma m}\left(E, E^{\prime}\right)= & \sum_{\gamma^{\prime} l^{\prime}}\left[\sum_{\gamma m, 12}^{r}(E) K_{\gamma \gamma^{\prime} l^{\prime}, m}\left(E^{\prime}\right)\right. \\
& \left.+\sum_{\gamma m, 12}^{<}(E) Q_{\gamma^{\prime} l^{\prime} m+\left[\mu_{\gamma^{\prime} \gamma} / \hbar \omega\right], 21}^{(+) a}\left(E^{\prime}\right)\right], \\
K_{\gamma \gamma^{\prime} l^{\prime}, m}(E)= & Q_{\gamma^{\prime} l^{\prime} m+[\mu}^{(+)<\gamma_{\gamma^{\prime} \gamma^{\prime \hbar \omega}, 21}(E)} \\
& \left.+\sum_{\gamma^{\prime \prime} l^{\prime \prime} m^{\prime}} Q_{\gamma^{\prime} l^{\prime} m^{\prime}, 22}^{(+)<}(E) Q_{\gamma^{\prime \prime} l^{\prime \prime} m+[\mu}^{(+) a} \mu_{\gamma^{\prime \prime} \gamma} / \hbar \omega\right], 21
\end{aligned}
$$

This Green function has the similar factorized form as the system with a central regime coupled with normal leads. ${ }^{20}$ If the two leads are normal metals, the energy gap $\Delta=0$, and we have normal Green functions by letting $Q_{\gamma l m, \alpha \beta}^{( \pm) X}(E)=0$ for $(\alpha \neq \beta)$ in Eqs. (15) and (18). Up to now, we have found the required Green functions for our system.
We can find the dc current by taking the time average over Eq. (9) and then obtain the formula expressed by the Fourier transformed normal Green function $G_{\sigma, \alpha \alpha}^{X}(E)$ as

$$
I_{L}=-\frac{2 e}{h} \operatorname{Im} \sum_{\sigma} \int d \epsilon \Gamma_{L}\left[f(\epsilon) G_{\sigma, 11}^{r}(\epsilon)+\frac{1}{2} G_{\sigma, 11}^{<}(\epsilon)\right] .
$$

Substituting the Green functions given in Eqs. (15) and (18) into the current formula above, one immediately arrives at the time-averaged tunneling current

$$
I=I_{(1)}+I_{(2)}+I_{(3)},
$$

where $I_{(1)}, I_{(2)}, I_{(3)}$ are the current components defined as follows. $I_{(1)}$ is the current given by

$$
I_{(1)}=\frac{e}{h} \sum_{m \sigma} \int d \epsilon T_{m, \sigma}^{(1)}(\epsilon)[f(\epsilon-e V)-f(\epsilon-m \hbar \omega)],
$$

where

$$
\begin{gathered}
T_{m, \sigma}^{(1)}(\epsilon)=J_{m}^{2}\left(\Lambda_{R L}\right) \Gamma_{L} \Gamma_{R} A_{\sigma}(\epsilon-e V) \\
\quad \times\left[\operatorname{Re} N_{R}(\epsilon-m \hbar \omega)+\eta_{m}^{A}(\epsilon)\right], \\
\eta_{m}^{A}(\epsilon)=-\frac{1}{4} J_{m}^{2}\left(\Lambda_{R L}\right) \Gamma_{R}\left|W_{R}(\epsilon-m \hbar \omega)\right|^{2} B_{\sigma}(\epsilon+e V \\
-2 m \hbar \omega) \operatorname{Re} \tau^{(+)}(\epsilon), \\
B_{\sigma}(\epsilon)=\frac{\Lambda_{\sigma}^{(+) 2}(\epsilon)}{\left[1-\operatorname{Re} \zeta_{\sigma}^{(+) r}(\epsilon)\right]^{2}+\left[\operatorname{Im} \zeta_{\sigma}^{(+) r}(\epsilon)\right]^{2}}, \\
W_{R}(\epsilon)=\frac{|\Delta|}{\left(\epsilon^{2}-|\Delta|^{2}\right)^{1 / 2}},
\end{gathered}
$$

$$
A_{\sigma}(\epsilon)=\frac{\Lambda_{\sigma}^{(-) 2}(\epsilon)}{\left\{1-\operatorname{Re}\left[\zeta_{\sigma}^{(-) r}(\epsilon)+\Pi_{\sigma}^{r}(\epsilon)\right]\right\}^{2}+\left\{\operatorname{Im}\left[\zeta_{\sigma}^{(-) r}(\epsilon)+\Pi_{\sigma}^{r}(\epsilon)\right]\right\}^{2}}
$$

In the current component $I_{(1)}$ there exists two processes of reflection: the normal reflection and the Andreev reflection. As $\Delta \rightarrow 0$, we obtain the normal current of the system without the superconducting threshold, and hence there is no Andreev reflection. The term containing $\eta_{m}^{A}(\epsilon)$ contributes the Andreev tunneling current in the formula, Eq. (21). However, since the normal tunneling current involves the density of state $\operatorname{Re} N_{R}(\epsilon)$, this normal current is zero as $\mid e V$ $+m \hbar \omega|<| \Delta \mid$ at zero temperature, while the term containing $\eta_{m}^{A}(\epsilon)$ has a contribution in this region, but with different resonant behaviors. This Andreev tunneling current is nonzero as $|e V+m \hbar \omega|>|\Delta|$, but it is much smaller than the normal current in this region for the weakly coupled system.
The current component $I_{(2)}$ is determined by the formula

$$
\begin{aligned}
I_{(2)}= & \frac{e}{h} \sum_{m \sigma} \int d \epsilon T_{m, \sigma}^{(2)}(\epsilon)[f(\epsilon-m \hbar \omega) \\
& -f(\epsilon+e V-2 m \hbar \omega)]
\end{aligned}
$$

where $T_{m, \sigma}^{(2)}(\epsilon)=\Theta_{m, \sigma}(\epsilon) \Gamma_{L}^{2}$, and

$$
\begin{aligned}
\Theta_{m, \sigma}(\epsilon)= & \frac{1}{4} J_{m}^{4}\left(\Lambda_{R L}\right)\left|\Gamma_{R} W_{R}(\epsilon-m \hbar \omega)\right|^{2} \\
& \times B_{\sigma}(\epsilon+e V-2 m \hbar \omega) A_{\sigma}(\epsilon-e V) .
\end{aligned}
$$


In the tunneling current, the factors $A_{\sigma}(\epsilon)$ and $B_{\sigma}(\epsilon)$ are involved, and they provide resonant peaks whose positions $\epsilon_{i}$ satisfy the equations $\left(\epsilon_{i}+\widetilde{E}_{d, l \sigma}+\sigma \mu B\right)^{2}-\operatorname{Re}\left[\zeta_{\sigma}^{(+) r}\left(\epsilon_{i}\right)\right]$ $=0$, and $\left(\epsilon_{i}-\widetilde{E}_{d, l \sigma}+\sigma \mu B\right)^{2}-\operatorname{Re}\left[\zeta_{\sigma}^{(-) r}\left(\epsilon_{i}\right)+\Pi_{\sigma}^{r}\left(\epsilon_{i}\right)\right]=0$. Equations (21) and (22) become zero as the ac field and source-drain dc biases are removed. This term is a part of the Andreev tunneling current induced by the Andreev reflection. It contributes to the Andreev current in the whole energy region. In the region $|e V+m \hbar \omega|<|\Delta|$, the normal tunneling is zero, and the Andreev current takes a major effect. Equation (22) is zero as the energy gap $\Delta=0$, i.e., for the system coupled with two normal leads, there is no current term.

The current component $I_{(3)}$ is determined by

$$
\begin{aligned}
I_{(3)}= & \frac{e}{h} \sum_{m m^{\prime} \sigma} \int d \epsilon T_{m m^{\prime}, \sigma}^{(3)}(\epsilon)\left\{f\left(\epsilon-m^{\prime} \hbar \omega\right)\right. \\
& \left.-f\left[\epsilon+\left(m-2 m^{\prime}\right) \hbar \omega\right]\right\},
\end{aligned}
$$

where

$$
T_{m m^{\prime}, \sigma}^{(3)}(\epsilon)=\Theta_{m^{\prime}, \sigma}(\epsilon) J_{m}^{2}\left(\Lambda_{R L}\right) \Gamma_{L} \Gamma_{R} .
$$

Equation (23) is a compound effect caused by the microwave field and the Andreev reflection. This part of the Andreev current is ascribed to the photon-electron pumping behavior, and it becomes zero as the ac field is removed from the system. The procedure of transport can be understood as the quasiparticles tunnel from the left lead to the right junction undertaking the Andreev reflection at the right barrier. This Andreev reflection is associated with the photon absorption and emission. Obviously, this part of the current disappears as the two leads are normal.

From the formula (20) one sees that the current is composed of normal and Andreev tunnelings. As the energy gap $\Delta \rightarrow 0$, the Andreev current disappears, and we obtain the Landauer-Büttiker-like formula of the normal system with the transmission probability $T_{m, \sigma}^{(1)}(\epsilon)$ $=J_{m}^{2}\left(\Lambda_{R L}\right) \Gamma_{L} \Gamma_{R} A_{\sigma}(\epsilon)$. The normal tunneling possesses Breit-Wigner resonance, while for the Andreev tunneling this resonance is broken. The Andreev current can be induced by the dc as well as ac fields. If the dc source-drain bias is zero, we still have the Andreev tunneling current caused by the ac field. The Andreev reflection can induce additional resonant peaks in the region where the normal tunneling is zero. On the other hand, the Zeeman field does not drive the charge carriers to form a tunneling current. But it splits the resonant levels to form a nondegenerated system, and the current oscillates in the split channels.

\section{NUMERICAL RESULTS AND ANALYSES}

In this section we perform the numerical calculations on the tunneling current at zero temperature. We take the photon energy $\hbar \omega$ of the external ac field as the energy scale of measurement. So all the energy quantities of the system can be expressed by using the photon energy as a measurement unit, such as $|\Delta|=p \hbar \omega$. In the numerical calculations, we denote the absolute value of the energy gap $|\Delta|$ to be $\Delta$ for

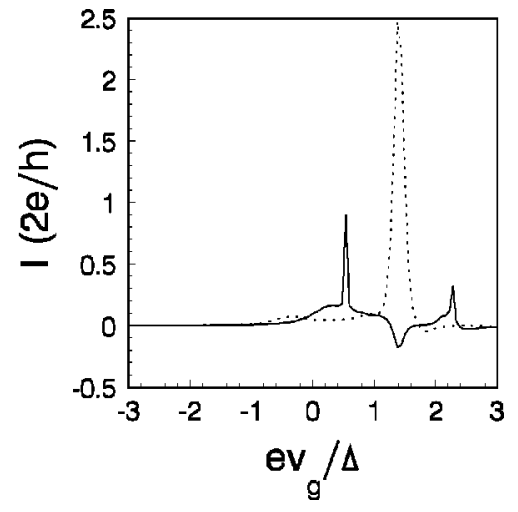

FIG. 1. The resonant current versus gate voltage $v_{g}$ for the single-channel system in the absence of the ac field. The parameters are chosen as $\Gamma=0.2 \Delta, E_{d}=0.2 \Delta, e V=1.2 \Delta$, and for the solid curve $\mu B=0$; for the dotted curve $\mu B=0.6 \Delta$.

convenience. At zero temperature, the Fermi distribution function is $f(\epsilon)=1-\theta(\epsilon)$, where $\theta(\epsilon)$ is the step function. Therefore, the tunneling current (20) at zero temperature is reduced to

$$
\begin{aligned}
I= & \frac{e}{h} \sum_{m \sigma}\left\{\int_{0}^{e V-m \hbar \omega}\left[T_{m, \sigma}^{(1)}(\epsilon)+T_{m, \sigma}^{(2)}(-\epsilon)\right] d \epsilon\right. \\
& \left.-\sum_{m^{\prime}} \int_{0}^{\left(m-m^{\prime}\right) \hbar \omega} T_{m m^{\prime}, \sigma}^{(3)}(\epsilon) d \epsilon\right\} .
\end{aligned}
$$

We deal with the special case where the linewidths of the left and right leads are equal, i.e., $\Gamma_{L}=\Gamma_{R}=\Gamma$. We study the single-channel and multichannel systems separately in the following two subsections. We consider the microwave field to be located in the frequency regime $\omega=2 \pi \times 10^{11} \mathrm{~Hz}$. The photon energy of the microwave field is about $0.4 \mathrm{meV}$. In the numerical calculations, we assume that the energy gap of the superconducting lead is comparable with the photon energy. The materials having such energy gaps are $\mathrm{Ga}, \mathrm{Tl}$, and In with corresponding energy gaps of $0.165 \mathrm{meV}, 0.368 \mathrm{meV}$, and $0.52 \mathrm{meV}$, for instance.

\section{A. The single-channel system}

In this subsection, we consider the single-channel system where the energy of electrons in the quantum dot only have one level $E_{d}$. We show the tunneling behaviors versus gate voltage and source-drain bias by solving the current formula (24) numerically. The diameter of the quantum dot is on the order of $10 \mathrm{~nm}$.

We present the resonant structure of the tunneling current versus gate voltage in Fig. 1 as the microwave field is removed. The solid and dotted curves are associated with the situations in the absence and presence of the Zeeman magnetic field, respectively. The Andreev reflection has been taken into account in the evaluation. As the Zeeman magnetic field is removed from the quantum dot, two resonant peaks emerge, and the negative current is observed. As the magnetic field is applied to the quantum dot, the two peaks disappear and a large resonant peak appears. The negative 


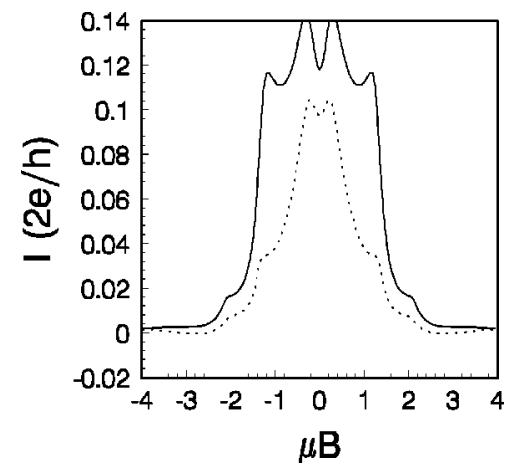

FIG. 2. The tunneling current versus Zeeman energy $\mu B$ for the single-channel system. The parameters are chosen as $\Gamma=0.2 \hbar \omega$, $\Delta=\hbar \omega, e V=1.2 \hbar \omega, E_{d}=0.2 \hbar \omega$, and for the dotted curve $\Lambda_{R L}$ $=0.3$; for the solid curve $\Lambda_{R L}=0.8$.

valley and lower resonant peak located at $2.3 \Delta$ are caused by the Andreev reflection. The Zeeman magnetic field raises the negative valley to the height $I=5.0 \mathrm{e} / \mathrm{h}$, and the two resonant peaks are suppressed. The negative valley is also suppressed and shifted by the Zeeman magnetic field.

Figure 2 displays the resonant behaviors of the tunneling current versus Zeeman energy $\mu B$. The different curves indicate the influence of the ac field with different magnitudes on the tunneling current. The dotted curve represents the situation where $\Lambda_{R L}=0.3$, and the solid curve is for $\Lambda_{R L}$ $=0.8$. The current is sensitive to the magnitude of the ac field. As the ac field is weak, a negative tunneling current is observed around $|\mu B| \sim 2.5 \hbar \omega$. The side steps signify photon absorption and emission of transporting electrons. As the magnitude of the ac field becomes large, the current rises abruptly, and a resonant structure appears. The negative current disappears, and the maximum height of the resonant peaks is larger than the one applied by the weak ac field. The structure is symmetric about the Zeeman energy.

We present the $I-V$ characteristics of the system in Fig. 3 to show the Zeeman splitting of the photon-assisted tunneling. In the absence of the ac field the tunneling current is zero as $|e V|<\Delta$ by neglecting the Andreev current. As the ac field is applied, there exists a tunneling current due to the charging-discharging effect. This charging-discharging cur-

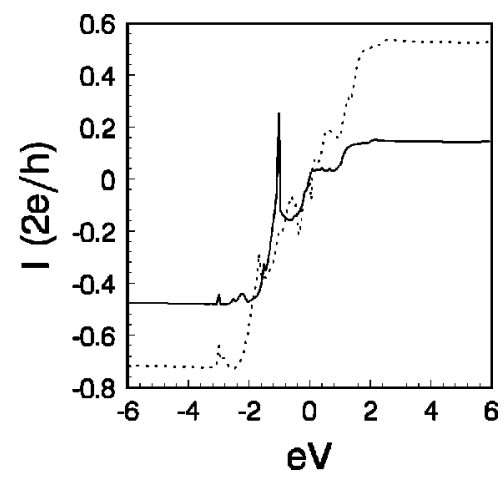

FIG. 3. The $I-V$ characteristics of the single-channel system in the presence of a microwave field. The parameters are chosen as $\Gamma=0.2 \hbar \omega, \Delta=\hbar \omega, \Lambda_{R L}=0.8, E_{d}=0.2 \hbar \omega$, and for the solid curve $\mu B=0$; for the dotted curve $\mu B=0.6 \hbar \omega$. rent is nonzero by taking the time average. The energy gap of the superconducting lead acts as a barrier for electrons to transport. As the source-drain voltage $V$ lies in the regime $0<e V<\Delta$, the electrons are accelerated by the voltage, and a small part of them can tunnel through the barrier due to the Andreev reflection. As the ac field is applied, the electrons absorb photon energy to raise their potentials, and we may have the relation $|e V+m \hbar \omega|>\Delta$. So these electrons can overcome the threshold $\Delta$ to form a normal tunneling current. This effect is known as the photon-electron pumping effect in the NDS system. On the other hand, the electrons may lose their energy by emitting photons and drop to lower potential levels. Thus, the electrons cannot transport through the quantum-dot regime even if their original accelerated energy is larger than the energy gap. This procedure can be described as the situation where the source-drain voltage provides energy for electrons to meet $|e V|>\Delta$, but some of the energy changes into photon energy to meet $|e V+m \hbar \omega|<\Delta$. However, the Andreev reflection can take place in the regime $0<|e V+m \hbar \omega|<\Delta$. So the tunneling current for this situation contains the compound effect of photon-assisted Andreev tunneling (PAAT). ${ }^{18}$ The solid curve denotes PAAT current-voltage behavior in the absence of the Zeeman magnetic field. The usual steps in the normal system are modified by superimposing some Andreev tunneling peaks on the steps and plateau. The dotted curve represents the PAAT current split by the Zeeman magnetic field. The tunneling current stretches up and down, and the absolute value of the saturated tunneling current becomes larger. Each step is split and the Andreev reflection peak is suppressed by the Zeeman magnetic field. For the ac field applied system, the photon energy forms sidebands of electrons, and the Zeeman field splits each sideband of electrons in the quantum dot to form multichannel $E_{d}+m \hbar \omega+\sigma \mu B$ for electrons to tunnel. Therefore, we can obtain a larger current and rich physical features for the tunneling current by applying the Zeeman magnetic field.

\section{B. The multichannel system}

In this subsection we perform the numerical calculations on the tunneling current through the multichannel quantumdot system. We assume the quantum dot possesses five levels with equal energy spacing, i.e., $E_{d l}=l \Delta E_{d}$, where $\Delta E_{d}$ $=0.5 \hbar \omega$, and $l=1, \ldots, 5$.

Figure 4 represents the resonant behaviors of the tunneling current versus gate voltage for the multichannel system. Diagram (a) denotes the situation without applying the Zeeman magnetic field. A negative valley is located in the central regime of the resonant peaks. Since the resonant peaks rise erectly, the photon-assisted side peaks are not obviously visible. The heights of the peaks are not equal, which shows the asymmetric behavior of the tunneling current with respect to the gate voltage. Diagram (b) indicates the Zeeman splitting of the resonant structure in the current. Each of the resonant peaks is split to form a nondegenerated tunneling current. The negative valley is also split to form four negative ones. Comparing the two cases, we observe that the magnetic field can suppress the magnitude of the tunneling current, and it provides split channels for electrons to tunnel. 


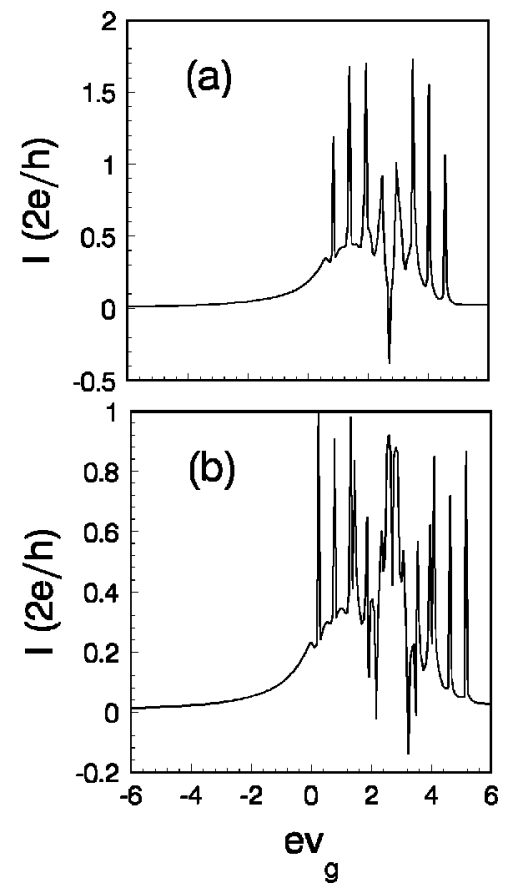

FIG. 4. The tunneling current versus gate voltage for the multichannel system. The parameters are chosen as $\Gamma=0.2 \hbar \omega, \Lambda_{R L}$ $=0.8, \Delta=\hbar \omega, l=5$, and for diagram (a) $\mu B=0$; for diagram (b) $\mu B=0.6 \hbar \omega$.

We display the current versus Zeeman energy in Figs. 5 and 6 . The tunneling current is symmetric with respect to the Zeeman energy for the system without Coulomb interaction. Positive resonant peaks and negative valleys emerge in the

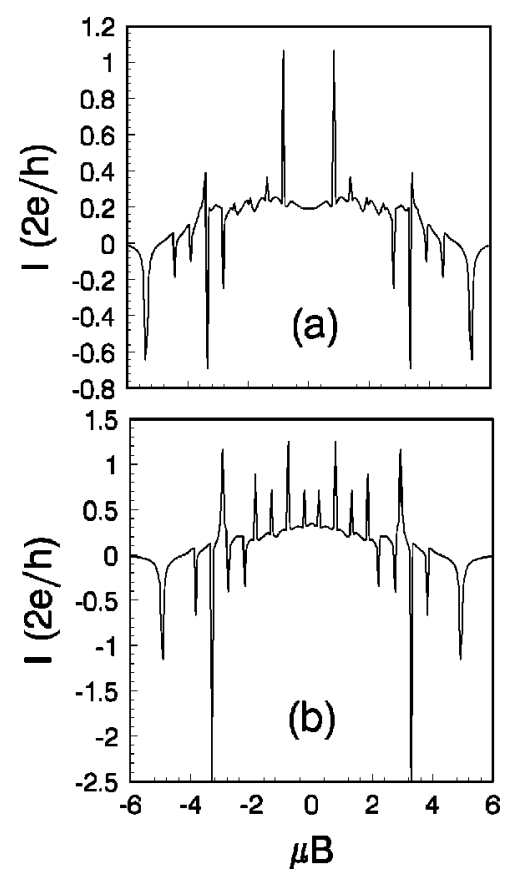

FIG. 5. The tunneling current versus Zeeman energy $\mu B$ for the multichannel system. The parameters are chosen as $\Gamma=0.2 \hbar \omega$, $\Lambda_{R L}=0.8, \Delta=\hbar \omega, e V=1.2 \hbar \omega$, and for diagram (a) $e v_{g}=0$; for diagram (b) $e v_{g}=0.6 \hbar \omega$.

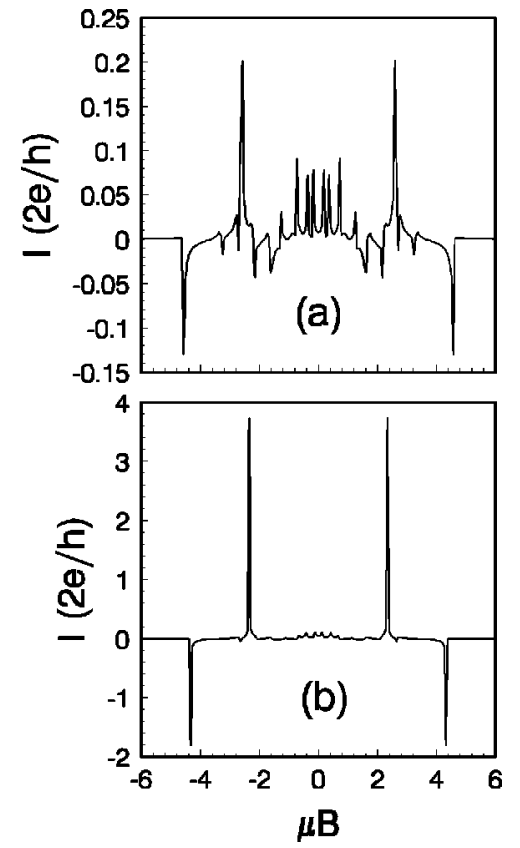

FIG. 6. The tunneling current versus Zeeman energy $\mu B$ for the multichannel system. The parameters are chosen as those in Fig. 5 with respect to diagrams (a) and (b) except in this figure $e V=0$.

symmetric form about $\mu B=0$. Figure 5 shows the resonant behavior as the source-drain bias $V=1.2 \hbar \omega / e$. Diagrams (a) and (b) in this figure are depicted for the cases with gate voltage $v_{g}=0$ and $v_{g}=0.6 \hbar \omega / e$, respectively. The resonant structure is sensitive to gate voltage. Many resonant peaks appear as the gate voltage is applied to the quantum dot, and the magnitude of them increases due to the effect of $v_{g}$. The tunneling current structure is quite different from the one as the source-drain bias is removed (Fig. 6). The fine resonant structure is observed as the gate voltage is zero, and even peaks and valleys are restricted in the two side resonant peaks. As the gate voltage is applied, the two main positive peaks and negative side valleys increase their magnitudes. The central resonant peaks are small compared with the main peaks and valleys. In fact, the diagrams in Fig. 6 are associated with the photon-electron pumping effect, since the source-drain bias is zero. The electrons are pumped by a microwave field to overcome the superconducting barrier and to form a tunneling current. The electrons are also accelerated by the photon energy between the source and drain of the system. So this current contains a photon-electron pumping current and the Andreev tunneling current.

\section{CONCLUDING REMARKS}

We have investigated the tunneling current versus gate voltage, Zeeman energy, and source-drain bias in the NDS system applied with the ac microwave field. The main results are derived from the current formula given by Eq. (20). The current formula contains photon-assisted tunneling and Andreev reflection effects. The Zeeman effect is also hidden in Eq. (20). The tunneling current is zero as the source-drain bias and ac field are removed. This means that for the NDS 
system, the current is driven by the dc and ac fields. The current formula in this system is dominated by the DOS of the superconductor, and the current has a resonant structure deviating from the usual Breit-Wigner form. We have considered the Andreev reflection, which provides tunneling current even if the source-drain bias is zero for the photonassisted tunneling. We have performed the numerical calculation at zero temperature by using Eq. (24). The singlechannel and multichannel tunneling currents are evaluated separately, and the Zeeman-splitting current is discussed. The Zeeman-split PAAT resonant structures are obtained versus gate voltage and Zeeman energy. The tunneling current resonates in quite different ways with respect to the gate voltage and Zeeman energy. The tunneling resonant structure is symmetric versus Zeeman energy, while it is asymmetric versus gate voltage. Negative tunneling current is revealed both versus gate voltage and Zeeman energy. For the multichannel quantum-dot system we find that the tunneling structure versus Zeeman energy is very sensitive to gate voltage and source-drain bias. In the Andreev reflection regime, we may also obtain a large tunneling current by adjusting the
Zeeman energy and gate voltage. The Zeeman-split $I-V$ characteristics are presented for the single-channel system, and we observe that the Zeeman magnetic field may suppress the Andreev tunneling peaks. It splits and stretches the current to form large saturated values and current steps. From the above investigation we conclude that the Zeeman magnetic field can provide very interesting tunneling structures. Since we have several external parameters, such as gate voltage, source-drain bias, ac microwave field, and Zeeman magnetic field, we can obtain the desired tunneling current through the NDS system by adjusting the external parameters. These features may provide useful information for designing quantum devices.

\section{ACKNOWLEDGMENTS}

This work was supported by a RGC grant from the SAR Government of Hong Kong under Grant No. HKU 7215/99P, by the National Natural Science Foundation of China under Grant No. 19875004, and by the Associate Scheme of Abdus Salam International Center for Theoretical Physics.
*Mailing address: Department of Physics, Beijing Institute of Technology, Beijing 100081, China.

${ }^{1}$ D.C. Ralph, C.T. Black, and M. Tinkham, Phys. Rev. Lett. 74, 3241 (1995).

${ }^{2}$ J.v. Delft, A.D. Zaikin, D.S. Golubev, and W. Tichy, Phys. Rev. Lett. 77, 3189 (1996).

${ }^{3}$ T.M. Eiles, J.M. Martinis, and M.H. Devoret, Phys. Rev. Lett. 70, 1862 (1993).

${ }^{4}$ J.H. Xu, J.H. Miller, and C.S. Ting, Phys. Rev. B 53, 3604 (1996).

${ }^{5}$ M. Hurd, S. Datta, and P.F. Bagwell, Phys. Rev. B 54, 6557 (1996); H.T. Ilhan, H.V. Demir, and P. Bagwell, ibid. 58, 15120 (1998)

${ }^{6}$ H. Takayanagi, T. Akazaki, and J. Nitta, Phys. Rev. Lett. 75, 3533 (1995)

${ }^{7}$ A.F. Andreev, Zh. Éksp. Teor. Fiz. 46, 1823 (1964) [ Sov. Phys. JETP 19, 1228 (1964)]; G.E. Blonder, M. Tinkham, and T.M. Klapwijk, Phys. Rev. B 25, 4515 (1982).

${ }^{8}$ R. Kümmel, and W. Senftinger, Z. Phys. B: Condens. Matter 59, 275 (1985); P.G. de Gennes, Superconductivity of Metals and Alloys (Addison-Wesley, New York, 1989).

${ }^{9}$ D. Averin and A. Bardas, Phys. Rev. Lett. 75, 1831 (1995).

${ }^{10}$ M.J.M. De Jong and C.W.J. Beenakker, Phys. Rev. Lett. 74, 1657 (1995).
${ }^{11}$ U. Gunsenheimer and A.D. Zaikin, Phys. Rev. B 50, 6317 (1994); A.I. Larkin and Yu.N. Ovchinikov, Zh. Éksp. Teor. Fiz. 68, 1915 (1975) [ Sov. Phys. JETP 41, 960 (1976)].

${ }^{12}$ A.D. Zaikin, Zh. Éksp. Teor. Fiz. 84, 1560 (1983) [ Sov. Phys. JETP 57, 910 (1983)].

${ }^{13}$ S. Datta, P.F. Bagwell, and M.P. Anantram, Phys. Low-Dimens. Semicond. Struct. 3, 1 (1996); M.P. Samanta and S. Datta, Phys. Rev. B 57, 10972 (1998).

${ }^{14}$ F. Braun, J.v. Delft, D.C. Ralph, and M. Tinkham, Phys. Rev. Lett. 79, 921 (1997).

${ }^{15}$ K.M.H. Lenssen, M.R. Leys, and J.H. Wolter, Phys. Rev. B 58, 4888 (1998).

${ }^{16}$ N.R. Claughton, M. Leadbeater, and C.J. Lambert, J. Phys.: Condens. Matter 7, 8757 (1995).

${ }^{17}$ H.K. Zhao and G.v. Gehlen, Phys. Rev. B 58, 13660 (1998); H.K. Zhao, Phys. Lett. A 264, 218 (1999); 272, 197 (2000).

${ }^{18}$ Q.F. Sun, J. Wang, and T.H. Lin, Phys. Rev. B 59, 3831 (1999); 59, 13126 (1999).

${ }^{19}$ M. Tinkham, Introduction to Superconductivity (McGraw-Hill, New York, 1996).

${ }^{20}$ A.P. Jauho, N.S. Wingreen, and Y. Meir, Phys. Rev. B 50, 5528 (1994). 